\title{
A Smart Mobile Handset Plastic Case With Integrated Split Ring Resonators to Reduce SAR
}

\author{
Hengyi Zhou, Arpan Pal, Amit Mehta \\ College of Eng, Swansea University, Swansea, UK \\ 686785@swansea.ac.uk; a.pal@swansea.ac.uk. \\ a.mehta@swansea.ac.uk
}

\begin{abstract}
Majority of mobile phones use GSM band (900 and $1800 \mathrm{GHz})$, GPS band (1.5 GHz) and WiFi band (2.4 GHz). However, most modern mobile handsets can also communicate at another WiFi band which is the $5 \mathrm{GHz}$ band. A plastic smart case with three integrated Split Ring Resonators (SRR) is proposed to reduce SAR (Specific Absorption Rate) of a mobile handset at 5 $\mathrm{GHz}$ by mounting this case onto the phone. The radiated EM (Electro-Magnetic) field from the handset antenna interacts with the SRR layer and re-radiates into the far-field. SRR layer directs the radiation away from the handset screen and hence it reduces the human head exposure to the EM field during a call. It is observed that the smart case can reduce the $10 \mathrm{~g} \mathrm{SAR}$ from $2.49 \mathrm{~W} / \mathrm{kg}$ to $1.78 \mathrm{~W} / \mathrm{kg}(\approx 30 \%$ reduction) to comply with the SAR limit guideline.
\end{abstract}

Keywords-Split ring resonator, radiation pattern, SAR reduction.

\section{INTRODUCTION}

With the rapid development in wireless communications the use of mobile handsets is ever growing and they have become an essential part of the modern human life. As the usage of mobile handsets is increasing every year, the research on health risk from hazardous EM field emitted by mobile handsets is currently gaining much attention. The Specific Absorption Rate (SAR) is a defined parameter to evaluate the power absorption in human tissue. Safety guidelines in terms of SAR have been issued to reduce the exposure of the human tissues to EM fields [1]. For the mobile handsets, the SAR value must not exceed the maximum limits of the guidelines.

Various methods have been investigated to reduce the SAR of mobile handsets. A ferrite sheet [2], a thin metal shim-layer [3], a metamaterial layer consisting of Split Ring Resonators (SRR) [4]-[5] are applied between the handset antenna and human head for SAR reduction. These techniques have been implemented for GSM (900 and $1800 \mathrm{MHz}$ ) and $802.11 \mathrm{~b} / \mathrm{g} / \mathrm{n}$ WiFi $(2.45 \mathrm{GHz})$ frequency bands and they essentially would reduce the Total Radiated Power (TRP) from handsets in order to limit EM field exposure. Reduction in TRP adversely affect the signal reception. Most modern handsets are also capable of communication in $5 \mathrm{GHz}$ frequency band (IEEE-802.11a) for indoor $\mathrm{WiFi}$ applications. Hence, it is desirable for these handsets to have an SAR value fulfilling the guideline without affecting the signal reception.

In this paper, a plastic smart case containing three SRRs is proposed to reduce the $10 \mathrm{~g}$ SAR in $5 \mathrm{GHz}$ frequency band. The layer of SRR acts as a metamaterial [6] and enables the handsets to direct the beam away from the human head while a

\author{
Dariush Mirshekar-Syahkal \\ School of Computer Sc and Electronic Eng. \\ University of Essex, Colchester, UK \\ dariush@essex.co.uk
}

call is made. Thus, using this case the handsets are able to reduce the EM field exposure to the human head and reduce the SAR. A dipole antenna along with a mobile handset body is used to mimic the characteristics of most commercial handsets at $5 \mathrm{GHz}$. All the results presented in this paper are obtained using CST microwave studio, which is based on Finite Integration Technique in Time Domain (FIT-TD).

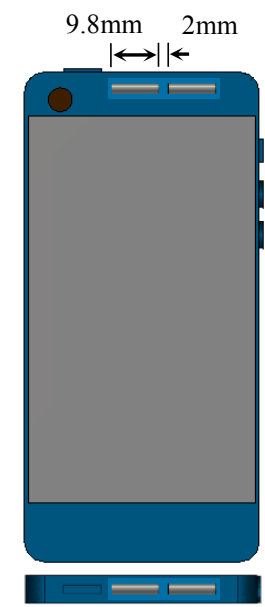

(a)

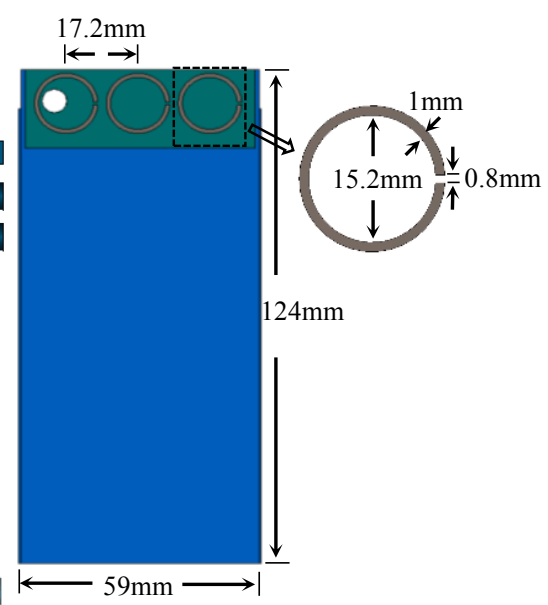

(b)
Fig. 1. (a) WiFi diplole antenna placement in a cellular phone (back view and top view of the model). (b) Proposed SRR phone case parameter details.

\section{HANDSET AND SMART CASE CONFIGURATION}

Fig. 1(a) shows the dipole antenna enclosed in a plastic housing and the complete handset model. The antenna has a total length of $21.6 \mathrm{~mm}$. The handset housing has a dimension of $124 \mathrm{~mm} \times 59 \mathrm{~mm} \times 7 \mathrm{~mm}$, which is in line with the standard of modern smartphones. The housing is designed with a plastic having a relative permittivity ( $(\varepsilon r)$ of 2.5 and conductivity $(\sigma)$ of $0.02 \mathrm{~S} / \mathrm{m}$. The diagonal dimension of the screen is $101.6 \mathrm{~mm}$ (4 inch) and the phone has a metal sheet behind the Liquid Crystal Display (LCD) in commercial handsets.

Fig. 1(b) shows the smart case with the SRR layer. Three Circular SRRs (CSRR) are implemented in the case. When the case is applied with the handset, the SRR layer appears on top of the antenna. The unit cell of the SRR structure consists of a circular ring having a diameter of $15.2 \mathrm{~mm}$ and a track width of $1 \mathrm{~mm}$. An air gap of $0.8 \mathrm{~mm}$ is inserted in the ring. Two neighbouring CSRR are placed $2 \mathrm{~mm}$ apart from each other. 


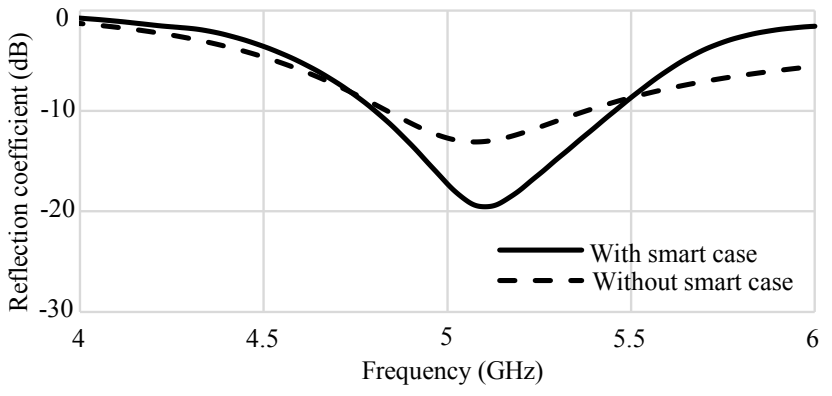

Fig. 2. Frequency response of reflection coefficient of the handset antenna with and without the smart case.

\section{REFLECTION COEFFICIENT AND RADIATION PATTERNS}

Fig. 2 shows the reflection coefficient of the antenna placed in the handset housing with and without the smart case. The antenna operates in the $5 \mathrm{GHz} \mathrm{WiFi}$ frequency band and offers an impedance bandwidth of $700 \mathrm{MHz}(4.7-5.4 \mathrm{GHz})$. The handset antenna combining with the smart case provides similar impedance bandwidth over the $5 \mathrm{GHz}$ band. However, due to the lower reflection coefficient the SRR layer enables the handset antenna to radiate more efficiently.

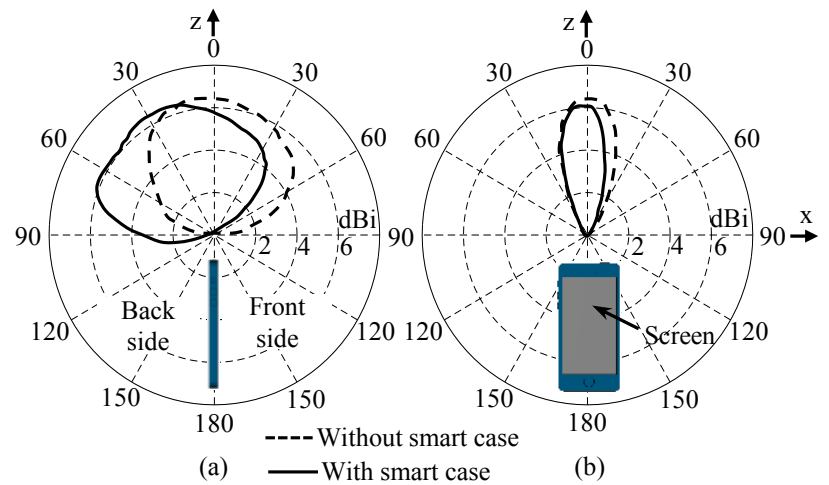

Fig. 3. SRR effect for antenna radiation pattern. (a) $\mathrm{phi}=0^{\circ}$, (b) $\mathrm{phi}=90^{\circ}$.

Fig. 3 shows the polar cuts (at $\phi=0^{\circ}$ and $\phi=90^{\circ}$ ) of radiation pattern of the handset with and without the smart case. The antenna together with the handset housing provides equal radiation in both front and back side of the handset. The maximum of the radiation occurs at $\theta_{\max }=0^{\circ}$. The antenna provides a gain of $6.3 \mathrm{dBi}$ with a radiation efficiency of $94.6 \%$. When the smart case is applied at the back side of the phone, the SRR layer act as metamaterial and alters the permittivity and permeability of the surface which enables the smart case to provide more EM wave transmission through the case. Consequently, the handsets with the case radiates more toward the backside away from the human head while making a phone call and maximum of the radiation occurs at $\theta_{\max }=-30^{\circ}$. The handset with the case offers a gain of $6.35 \mathrm{dBi}$ with a radiation efficiency of $96.3 \%$.

\section{SAR REDUCTION DUE TO SMART CASE}

The smart case reduces the front side radiation of handsets which lessens the human head to EM field exposure.
According to the recommendation made by the International Commission on Non-Ionising Radiation Protection (ICNIRP) guidelines [1], the European council has set a SAR limit of 2.0 $\mathrm{W} / \mathrm{kg}$ in $10 \mathrm{~g}$ of tissue. For the SAR calculation, the position of the handset is shown in the Fig. 4(a) and the input power is set to be $100 \mathrm{~mW}$. Fig. 4(b) and (c) show the SAR in human head due to the handset radiation with and without the smart case. When the handset is working independently it produces a maximum SAR of $2.498 \mathrm{~W} / \mathrm{kg}$ for $10 \mathrm{~g}$ averaging standard. However, the smart case enables the handset to reduce the maximum SAR to $1.784 \mathrm{~W} / \mathrm{kg}$ which is well under the safety limit.

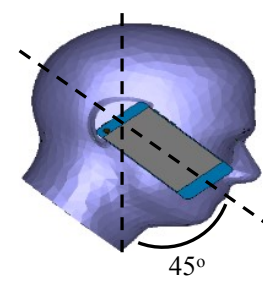

(a)

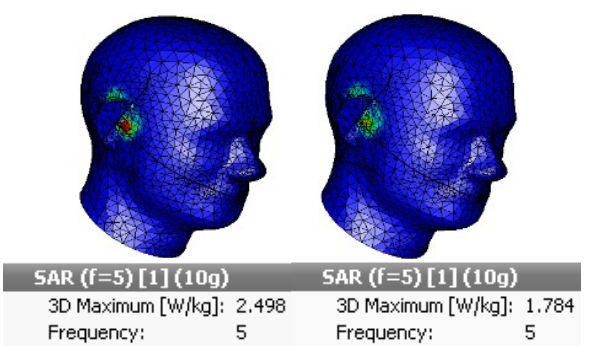

(b) (c)
Fig. 4. SAR distribution on a human model (a) due to handset (b) and due to handset combined with smart case (c).

\section{CONCLUSION}

A plastic smart case with three split ring resonators reduce the face side radiation of the mobile handset and reduce the EM field exposure of the human head while making a phone call. By using the smart case the maximum SAR of the human head can be reduced from $2.49 \mathrm{~W} / \mathrm{kg}$ to $1.78 \mathrm{~W} / \mathrm{kg}$ for $10 \mathrm{~g}$ averaging standard which is well below the maximum limit of the guideline.

\section{REFERENCES}

[1] International Non-Ionizing Radiation Committee of the InternationalRadiation Protection Association, "Guidelines on limits on exposure to radio frequency electromagnetic fields in the frequency range from $100 \mathrm{kHz}$ to $300 \mathrm{GHz}$," Health Physics, Vol. 54, No. I, pp. 115-123, 1988.

[2] J. Wang and O. Fujiwara, "Reduction of electromagnetic absorption in the human head for portable telephone by a ferrite sheet attachment," IEICE Trans. Communications, vol. E80B, no. 12, pp. 1810-1815, Dec. 1997.

[3] A. H. Kusuma, A.F. Sheta, I. M. Elshafiey, Z. Siddiqui, M. A. Alkanhal, S. Aldosari, "A new low SAR antenna structure for wireless handset applications," Prog Electromagn, vol. 112, pp. 23-40.

[4] J. Hwang, and F. Chen, "Reduction of the Peak SAR in the Human Head With Metamaterials," IEEE Trans. Antennas Propag., vol. 54, no. 12, pp. 3763-3770, Dec. 2006.

[5] M. I. Hossain, M.T. Islam, "Design of miniaturized double-negative material for specific absorption rate reduction in human head," PloS One, vol. 9, no. 10, oct. 2014.

[6] A. Lai, T. Itoh, and C. Caloz, "Composite right/left-handed transmission line metamaterials," IEEE Microw. Mag., vol. 5, no. 3, pp. 34-50, Sep. 2004. 